TUM-HEP-315/98

hep-ph/9806278

June 1998

\title{
Generalized Factorization in Non-leptonic Two-Body B-Decays: a Critical Look ${ }^{\prime \prime}$
}

\author{
Andrzej J. Buras and Luca Silvestrini \\ Technische Universität München, Physik Department \\ D-85748 Garching, Germany
}

\begin{abstract}
We reanalyze critically the generalized factorization hypothesis in non-leptonic twobody B-decays discussed recently by several authors. In particular we address the determination of the factorization scale $\mu_{f}$ and of the non-perturbative parameters $\xi_{1}^{\mathrm{NF}}\left(m_{\mathrm{b}}\right)$ and $\xi_{2}^{\mathrm{NF}}\left(m_{\mathrm{b}}\right)$ which are supposed to measure non-factorizable contributions to hadronic matrix elements with $\xi_{i}^{\mathrm{NF}}\left(\mu_{f}\right)=0$. We emphasize that both $\mu_{f}$ and $\xi_{i}^{\mathrm{NF}}\left(m_{\mathrm{b}}\right)$ are renormalization scheme dependent and we demonstrate analytically and numerically that for any chosen scale $\mu_{f}=\mathcal{O}\left(m_{\mathrm{b}}\right)$ it is possible to find a renormalization scheme for which $\xi_{1}^{\mathrm{NF}}\left(\mu_{f}\right)=\xi_{2}^{\mathrm{NF}}\left(\mu_{f}\right)=0$. The existing data indicate that such "factorization schemes" differ from the commonly used schemes NDR and HV. Similarly we point out that the recent extractions of the effective number of colours $N^{\mathrm{eff}}$ from two-body non-leptonic B-decays while $\mu$ and renormalization scheme independent suffer from gauge dependences and infrared dependences.
\end{abstract}

${ }^{*}$ Supported by the German Bundesministerium für Bildung und Forschung under contract 06 TM 874 and by the DFG project Li 519/2-2. 


\section{Introduction}

Two-body non-leptonic B-decays play an important role in the phenomenology of weak decays not only probing the structure of weak interactions corrected by short distance QCD effects but also providing some insight into the non-perturbative phenomena related to long distances. The increasing experimental information on these decays, in particular from the CLEO detector, stimulated recently several new theoretical analyses of these decays. The most extensive analyses of this type are based on the factorization of hadronic matrix elements of local operators [1]-[ [4] which has recently been extended to the so-called generalized factorization hypothesis [5]-[g].

In the strict factorization approach two-body decays are parametrized in terms of two phenomenological parameters $a_{1}$ and $a_{2}$ [3] which in QCD are given by

$$
a_{1}(\mu)=C_{1}(\mu)+\frac{1}{N} C_{2}(\mu), \quad a_{2}(\mu)=C_{2}(\mu)+\frac{1}{N} C_{1}(\mu) .
$$

Here $C_{1,2}(\mu)$ are the short distance Wilson coefficient functions of the relevant currentcurrent operators $O_{1,2}$ for which explicit expressions will be given below. $N$ is the number of colours with $N=3$ in QCD.

One distinguishes then three classes of decays for which the amplitudes have the following general structure [3, 团]:

$$
\begin{gathered}
A_{\mathrm{I}}=\frac{G_{F}}{\sqrt{2}} V_{C K M} a_{1}(\mu)\left\langle O_{1}\right\rangle_{F} \quad(\text { Class I) } \\
A_{\mathrm{II}}=\frac{G_{F}}{\sqrt{2}} V_{C K M} a_{2}(\mu)\left\langle O_{2}\right\rangle_{F} \quad(\text { Class II }) \\
A_{\mathrm{III}}=\frac{G_{F}}{\sqrt{2}} V_{C K M}\left[a_{1}(\mu)+x a_{2}(\mu)\right]\left\langle O_{1}\right\rangle_{F} \quad \text { (Class III) }
\end{gathered}
$$

Here $V_{C K M}$ denotes symbolically the CKM factor characteristic for a given decay. $\left\langle O_{i}\right\rangle_{F}$ are factorized hadronic matrix elements of the operators $O_{i}$ given as products of matrix elements of quark currents and $x$ is a non-perturbative factor equal to unity in the flavour symmetry limit.

The simplicity of this approach is very appealing. Once the matrix elements $\left\langle O_{i}\right\rangle_{F}$ have been expressed in terms of various meson decay constants and generally model dependent form factors, predictions for non-leptonic heavy meson decays can be made. An incomplete list of analyses of this type is given in [3, 4, 10] and will be extended below. 
On the other hand, it is well known that non-factorizable contributions must be present in the hadronic matrix elements of the current-current operators $O_{1}$ and $O_{2}$ in order to cancel the $\mu$ dependence of $C_{i}(\mu)$ or $a_{i}(\mu)$ so that the physical amplitudes do not depend on the arbitrary renormalization scale $\mu .\left\langle O_{i}\right\rangle_{F}$ being products of matrix elements of conserved currents are $\mu$-independent and the cancellation of the $\mu$ dependence in (2) -(4) does not take place. Consequently from the point of view of QCD the factorization approach can be at best correct at a single value of $\mu$, the so-called factorization scale $\mu_{f}$. Although the approach itself does not provide the value of $\mu_{f}$, the proponents of factorization expect $\mu_{f}=O\left(m_{b}\right)$ and $\mu_{f}=O\left(m_{c}\right)$ for B-decays and D-decays respectively.

The fact that $\left\langle O_{i}\right\rangle_{F}$ are $\mu$-independent but $a_{i}(\mu)$ are $\mu$-dependent, which is clearly inconsistent, inspired a number of authors [0]-9] to generalize the concept of factorization.

In the formulation due to Neubert and Stech [7] the $\mu$-dependent parameters $a_{1}(\mu)$ and $a_{2}(\mu)$ are replaced by $\mu$-independent effective parameters $a_{1}^{\text {eff }}$ and $a_{2}^{\text {eff }}$. The latter depend on $C_{i}(\mu)$ and two non-perturbative parameters $\varepsilon_{1}(\mu)$ and $\varepsilon_{8}(\mu)$ which parametrize the non-factorizable contributions to the hadronic matrix elements of the operators $O_{1,2}$. In the case of strict factorization $\varepsilon_{i}$ vanish and $a_{1,2}^{\text {eff }}$ reduce to $a_{1,2}(\mu)$. The $\mu$ dependence of $\varepsilon_{i}(\mu)$ cancels the $\mu$-dependence of $C_{1,2}(\mu)$ so that $a_{1,2}^{\mathrm{eff}}$ are indeed scale independent.

From the phenomenological point of view there is no change here relative to the standard factorization as only $a_{i}(\mu)$ have been replaced by $a_{i}^{\text {eff }}$ in the formulae (2)-(4). On the other hand, as stressed in [7], the new formulation should allow in principle some insight into the importance of non-factorizable contributions to hadronic matrix elements.

In this context we should remark that in the recent literature mainly the $\mu$ dependence of the non-factorizable contributions has been emphasized. Their renormalization scheme dependence has often not been discussed. It is the latter issue which will be important in the discussion below. Indeed at the next-to-leading level in the renormalization group improved perturbation theory the coefficients $C_{i}(\mu)$ depend on the renormalization scheme for operators. Again only the presence of non-factorizable scheme dependent contributions in $\left\langle O_{i}\right\rangle$ can remove this scheme dependence in the physical amplitudes and in particular in $a_{i}^{\text {eff }}$. The renormalization scheme dependence emphasized here, and discussed in the context of strict factoriza- 
tion in [11], is rather annoying from the factorization point of view as it precludes a unique phenomenological determination of $\mu_{f}$ as we will show explicitly below. In particular we will demonstrate that for any chosen scale $\mu_{f}=\mathcal{O}\left(m_{\mathrm{b}}\right)$ it is possible to find a renormalization scheme for which the non-factorizable parameters $\varepsilon_{1,8}\left(\mu_{f}\right)$ simultaneously vanish. This finding casts some doubts on the usefulness of the formulation in [7] with respect to the study of non-factorizable contributions to non-leptonic decays.

The generalized factorization presented in [5, 8, 9] is similar in spirit but includes more dynamics than the formulation in [7]. Here the non-factorizable contributions to the matrix elements are calculated in a perturbative framework at the one-loop level. Subsequently these non-factorizable contributions are combined with the coefficients $C_{i}(\mu)$ to obtain effective $\mu$ and renormalization scheme independent coefficients $C_{i}^{\text {eff }}$. The effective parameters $a_{i}^{\text {eff }}$ are given in this formulation as follows:

$$
a_{1}^{\mathrm{eff}}=C_{1}^{\mathrm{eff}}+\frac{1}{N^{\mathrm{eff}}} C_{2}^{\mathrm{eff}} \quad a_{2}^{\mathrm{eff}}=C_{2}^{\mathrm{eff}}+\frac{1}{N^{\mathrm{eff}}} C_{1}^{\mathrm{eff}}
$$

with analogous expressions for $a_{i}^{\text {eff }}(i=3-10)$ parametrizing penguin contributions. Here $N^{\text {eff }}$ is treated as a phenomenological parameter which models the nonfactorizable contributions to the hadronic matrix elements. In particular it has been suggested in [5, 8, 9] that the values for $N^{\text {eff }}$ extracted from the data on two-body non-leptonic decays should teach us about the pattern of non-factorizable contributions.

Unfortunately, as we will demonstrate below, also this approach has its weak points. Although $C_{1,2}^{\text {eff }}$ are $\mu$ and renormalization scheme independent, they are both gauge and infrared regulator dependent. The latter dependences originate in the perturbative evaluation of the scheme dependent finite contributions to the matrix elements, needed for the cancellation of the renormalization scheme dependence of $C_{i}(\mu)$. Consequently, whereas the extracted $N^{\text {eff }}$ is renormalization scheme and renormalization scale independent, it is a gauge and infrared regulator dependent quantity. This finding casts some doubts on the usefulness of the formulation in [5, 8, 9] with respect to the study of non-factorizable contributions to non-leptonic decays.

The rest of our paper amounts to putting all these statements in explicit terms. In section 2 we review the approach in [7]. In section 3 we reformulate this approach by replacing the parameters $\varepsilon_{1,8}(\mu)$ by two new parameters $\xi_{1,2}^{\mathrm{NF}}$. This reformulation allows us to make our points with regard to [7] in a more transparent manner. In 
particular we derive general expressions which allow to find, for a given $\mu_{f}$, the renormalization scheme in which $\varepsilon_{1,8}(\mu)$ or $\xi_{1,2}^{N F}(\mu)$ simultaneously vanish. In section 4 we illustrate our points with a few numerical examples and in section 5 we make a critical analysis of the approach in [5, 8, 9]. We end our paper with a brief summary and conclusions.

\section{Generalized Factorization}

In order to describe generalized factorization in explicit terms let us consider the decay $\bar{B}^{0} \rightarrow D^{+} \pi^{-}$. Then the relevant effective Hamiltonian is given by

$$
H_{\mathrm{eff}}=\frac{G_{F}}{\sqrt{2}} V_{c b} V_{u d}^{*}\left[C_{1}(\mu) O_{1}+C_{2}(\mu) O_{2}\right]
$$

where

$$
O_{1}=\left(\bar{d}_{\alpha} u_{\alpha}\right)_{V-A}\left(\bar{c}_{\beta} b_{\beta}\right)_{V-A}, \quad O_{2}=\left(\bar{d}_{\alpha} u_{\beta}\right)_{V-A}\left(\bar{c}_{\beta} b_{\alpha}\right)_{V-A}
$$

with $(\alpha, \beta=1,2,3)$ denoting colour indices and $V-A$ referring to $\gamma_{\mu}\left(1-\gamma_{5}\right) . C_{1}(\mu)$ and $C_{2}(\mu)$ are short distance Wilson coefficients computed at the renormalization scale $\mu=O\left(m_{b}\right)$. Note that we use here the labelling of the operators as given in [3, 4] which differs from [12 - 15] by the interchange $1 \leftrightarrow 2$. Since all four quark flavours entering the operators in (7) are different from each other, no penguin operators contribute to this decay.

Using Fierz reordering and colour identities one can rewrite the amplitude for $\bar{B}^{0} \rightarrow D^{+} \pi^{-}$as

$$
A\left(\bar{B}^{0} \rightarrow D^{+} \pi^{-}\right)=\frac{G_{F}}{\sqrt{2}} V_{c b} V_{u d}^{*} a_{1}^{\text {eff }}\left\langle O_{1}\right\rangle_{F}
$$

where

$$
\left\langle O_{1}\right\rangle_{F}=\left\langle\pi^{-}\left|(\bar{d} u)_{V-A}\right| 0\right\rangle\left\langle D^{+}\left|(\bar{c} b)_{V-A}\right| \bar{B}^{0}\right\rangle
$$

is the factorized matrix element of the operator $O_{1}$ and summation over colour indices in each current is understood.

The effective parameter $a_{1}^{\text {eff }}$ is given by [7]

$$
a_{1}^{\mathrm{eff}}=\left(C_{1}(\mu)+\frac{1}{N} C_{2}(\mu)\right)\left[1+\varepsilon_{1}^{(B D, \pi)}(\mu)\right]+C_{2}(\mu) \varepsilon_{8}^{(B D, \pi)}(\mu) .
$$

$\varepsilon_{1}^{(B D, \pi)}(\mu)$ and $\varepsilon_{8}^{(B D, \pi)}(\mu)$ are two hadronic parameters defined by $\llbracket$

$$
\varepsilon_{1}^{(B D, \pi)}(\mu) \equiv \frac{\left\langle\pi^{-} D^{+}\left|(\bar{d} u)_{V-A}(\bar{c} b)_{V-A}\right| \bar{B}^{0}\right\rangle}{\left\langle O_{1}\right\rangle_{F}}-1
$$


and

$$
\varepsilon_{8}^{(B D, \pi)}(\mu) \equiv 2 \frac{\left\langle\pi^{-} D^{+}\left|\left(\bar{d} t_{a} u\right)_{V-A}\left(\bar{c} t_{a} b\right)_{V-A}\right| \bar{B}^{0}\right\rangle}{\left\langle O_{1}\right\rangle_{F}}
$$

with $t_{a}$ denoting the colour matrices in the standard Feynman rules. $\varepsilon_{i}(\mu)$ parametrize the non-factorizable contributions to the hadronic matrix elements of operators. In the case of strict factorization $\varepsilon_{i}$ vanish.

It should be emphasized that no approximation has been made in (8). Since the matrix element $\left\langle O_{1}\right\rangle_{F}$ is scale and renormalization scheme independent this must also be the case for the effective coefficient $a_{1}^{\text {eff }}$. Indeed the scale and scheme dependences of the coefficients $C_{1}(\mu)$ and $C_{2}(\mu)$ are cancelled by those present in the hadronic parameters $\varepsilon_{i}(\mu)$. We will give explicit formulae for these dependences below.

A similar exercise with the amplitude for $\bar{B}^{0} \rightarrow D^{0} \pi^{0}$ gives

$$
A\left(\bar{B}^{0} \rightarrow D^{0} \pi^{0}\right)=\frac{G_{F}}{\sqrt{2}} V_{c b} V_{u d}^{*} a_{2}^{\text {eff }}\left\langle O_{2}\right\rangle_{F}
$$

where

$$
\left\langle O_{2}\right\rangle_{F}=\left\langle D^{0}\left|(\bar{c} u)_{V-A}\right| 0\right\rangle\left\langle\pi^{0}\left|(\bar{d} b)_{V-A}\right| \bar{B}^{0}\right\rangle
$$

is the factorized matrix element of the operator $\mathrm{O}_{2}$.

The effective parameter $a_{2}^{\text {eff }}$ is given by [7]

$$
a_{2}^{\mathrm{eff}}=\left(C_{2}(\mu)+\frac{1}{N} C_{1}(\mu)\right)\left[1+\varepsilon_{1}^{(B \pi, D)}(\mu)\right]+C_{1}(\mu) \varepsilon_{8}^{(B \pi, D)}(\mu) .
$$

$\varepsilon_{1}^{(B \pi, D)}(\mu)$ and $\varepsilon_{8}^{(B \pi, D)}(\mu)$ are two hadronic parameters defined by

$$
\varepsilon_{1}^{(B \pi, D)}(\mu) \equiv \frac{\left\langle\pi^{0} D^{0}\left|(\bar{c} u)_{V-A}(\bar{d} b)_{V-A}\right| \bar{B}^{0}\right\rangle}{\left\langle O_{2}\right\rangle_{F}}-1
$$

and

$$
\varepsilon_{8}^{(B \pi, D)}(\mu) \equiv 2 \frac{\left\langle\pi^{0} D^{0}\left|\left(\bar{c} t_{a} u\right)_{V-A}\left(\bar{d} t_{a} b\right)_{V-A}\right| \bar{B}^{0}\right\rangle}{\left\langle O_{2}\right\rangle_{F}}
$$

Again the $\mu$ and scheme dependences of $\varepsilon_{i}$ in (16) and (17) cancel the corresponding dependences in $C_{i}(\mu)$ so that the effective coefficient $a_{2}^{\text {eff }}$ is $\mu$ and scheme independent.

Following section 5.1 of [12] it is straightforward to find the explicit $\mu$ and scheme dependences of the hadronic parameters $\varepsilon_{i}(\mu)$. To this end we note that the $\mu$ dependence of the matrix elements of the operators $O_{ \pm}=\left(O_{1} \pm O_{2}\right) / 2$ is given by 12

$$
\left\langle O_{ \pm}(\mu)\right\rangle=U_{ \pm}\left(m_{\mathrm{b}}, \mu\right)\left\langle O_{ \pm}\left(m_{\mathrm{b}}\right)\right\rangle
$$


where the evolution function $U_{ \pm}\left(m_{\mathrm{b}}, \mu\right)$ including NLO QCD corrections is given by

$$
U_{ \pm}\left(m_{\mathrm{b}}, \mu\right)=\left[1+\frac{\alpha_{s}\left(m_{\mathrm{b}}\right)}{4 \pi} J_{ \pm}\right]\left[\frac{\alpha_{s}(\mu)}{\alpha_{s}\left(m_{\mathrm{b}}\right)}\right]^{d_{ \pm}}\left[1-\frac{\alpha_{s}(\mu)}{4 \pi} J_{ \pm}\right]
$$

with

$$
\begin{gathered}
J_{ \pm}=\frac{d_{ \pm}}{\beta_{0}} \beta_{1}-\frac{\gamma_{ \pm}^{(1)}}{2 \beta_{0}}, \quad d_{ \pm}=\frac{\gamma_{ \pm}^{(0)}}{2 \beta_{0}}, \\
\gamma_{ \pm}^{(0)}= \pm 2(3 \mp 1), \quad \beta_{0}=11-\frac{2}{3} f, \quad \beta_{1}=102-\frac{38}{3} f, \\
\gamma_{ \pm}^{(1)}=\frac{3 \mp 1}{6}\left[-21 \pm \frac{4}{3} f-2 \beta_{0} \kappa_{ \pm}\right] .
\end{gathered}
$$

Here $\kappa_{ \pm}$, introduced in [11], distinguishes between various renormalization schemes:

$$
\kappa_{ \pm}=\left\{\begin{array}{rcr}
0 & (\mathrm{NDR}) & {[16]} \\
\mp 4 & (\mathrm{HV}) & {[16} \\
\mp 6-3 & (\mathrm{DRED}) & 17
\end{array}\right.
$$

Thus $J_{ \pm}$in $(20)$ can also be written as

$$
J_{ \pm}=\left(J_{ \pm}\right)_{N D R}+\frac{3 \mp 1}{6} \kappa_{ \pm}=\left(J_{ \pm}\right)_{N D R} \pm \frac{\gamma_{ \pm}^{(0)}}{12} \kappa_{ \pm} .
$$

The $\overline{M S}$ coupling $[18]$ is given by

$$
\alpha_{s}(\mu)=\frac{4 \pi}{\beta_{0} \ln \left(\mu^{2} / \Lambda \frac{2}{M S}\right)}\left[1-\frac{\beta_{1}}{\beta_{0}^{2}} \frac{\ln \ln \left(\mu^{2} / \Lambda_{\overline{M S}}^{2}\right)}{\ln \left(\mu^{2} / \Lambda_{\overline{M S}}^{2}\right)}\right] .
$$

The formulae given above depend on $f$, the number of active flavours. In the case of B-decays $f=5$. The present world average for $\alpha_{s}\left(M_{Z}\right)$ is [19]:

$$
\alpha_{s}\left(M_{Z}\right)=0.118 \pm 0.003 \quad \Lambda \frac{(5)}{M S}=(225 \pm 40) \mathrm{MeV}
$$

where the superscript stands for $f=5$.

Having these formulae at hand it is straightforward to show that the $\mu$-dependence of $\varepsilon_{1}(\mu)$ and $\varepsilon_{8}(\mu)$ is governed by the following equations:

$$
\begin{aligned}
& 1+\varepsilon_{1}(\mu)= \frac{1}{2}\left[\left(1+\frac{1}{N}\right)\left[1+\varepsilon_{1}\left(m_{\mathrm{b}}\right)\right]+\varepsilon_{8}\left(m_{\mathrm{b}}\right)\right] U_{+}\left(m_{\mathrm{b}}, \mu\right) \\
&+ \frac{1}{2}\left[\left(1-\frac{1}{N}\right)\left[1+\varepsilon_{1}\left(m_{\mathrm{b}}\right)\right]-\varepsilon_{8}\left(m_{\mathrm{b}}\right)\right] U_{-}\left(m_{\mathrm{b}}, \mu\right), \\
& \varepsilon_{8}(\mu)=\frac{1}{2}\left[\left(1-\frac{1}{N}\right) \varepsilon_{8}\left(m_{\mathrm{b}}\right)+\left(1-\frac{1}{N^{2}}\right)\left[1+\varepsilon_{1}\left(m_{\mathrm{b}}\right)\right]\right] U_{+}\left(m_{\mathrm{b}}, \mu\right) \\
&+\frac{1}{2}\left[\left(1+\frac{1}{N}\right) \varepsilon_{8}\left(m_{\mathrm{b}}\right)-\left(1-\frac{1}{N^{2}}\right)\left[1+\varepsilon_{1}\left(m_{\mathrm{b}}\right)\right]\right] U_{-}\left(m_{\mathrm{b}}, \mu\right) .
\end{aligned}
$$


These formulae reduce to the ones given in [7] when $J_{ \pm}$in (19) are set to zero. They give both the $\mu$-dependence and renormalization scheme dependence of $\varepsilon_{i}$. The latter dependence has not been considered in [0]

\section{A Different Formulation}

In order to be able to discuss the relation of our work to the one of [7] we have used until now, as in [7], the hadronic parameters $\varepsilon_{1}(\mu)$ and $\varepsilon_{8}(\mu)$ to describe non-factorizable contributions. On the other hand, it appears to us that it is more convenient to work instead with two other parameters defined simply by

$$
a_{1}^{\mathrm{eff}}=a_{1}(\mu)+\xi_{1}^{\mathrm{NF}}(\mu), \quad a_{2}^{\mathrm{eff}}=a_{2}(\mu)+\xi_{2}^{\mathrm{NF}}(\mu)
$$

where $a_{1}(\mu)$ and $a_{2}(\mu)$ are given in (11).

Comparison with (10) and (15) gives

$$
\begin{aligned}
& \xi_{1}^{\mathrm{NF}}(\mu)=\varepsilon_{1}(\mu) a_{1}(\mu)+\varepsilon_{8}(\mu) C_{2}(\mu), \\
& \xi_{2}^{\mathrm{NF}}(\mu)=\bar{\varepsilon}_{1}(\mu) a_{2}(\mu)+\bar{\varepsilon}_{8}(\mu) C_{1}(\mu),
\end{aligned}
$$

where

$$
\begin{array}{ll}
\varepsilon_{1}(\mu)=\varepsilon_{1}^{(B D, \pi)}, & \varepsilon_{8}(\mu)=\varepsilon_{8}^{(B D, \pi)}, \\
\bar{\varepsilon}_{1}(\mu)=\varepsilon_{1}^{(B \pi, D)}, & \bar{\varepsilon}_{8}(\mu)=\varepsilon_{8}^{(B \pi, D)} .
\end{array}
$$

and $a_{i}(\mu)$, given in (11), are the parameters used in the framework of the strict factorization hypothesis in which $\xi_{i}^{\mathrm{NF}}(\mu)$ are set to zero. Their $\mu$ and renormalization scheme dependence can be studied using

$$
C_{1}(\mu)=\frac{z_{+}(\mu)+z_{-}(\mu)}{2}, \quad C_{2}(\mu)=\frac{z_{+}(\mu)-z_{-}(\mu)}{2}
$$

where

$$
z_{ \pm}(\mu)=\left[1+\frac{\alpha_{s}(\mu)}{4 \pi} J_{ \pm}\right]\left[\frac{\alpha_{s}\left(M_{W}\right)}{\alpha_{s}(\mu)}\right]^{d_{ \pm}}\left[1+\frac{\alpha_{s}\left(M_{W}\right)}{4 \pi}\left(B_{ \pm}-J_{ \pm}\right)\right]
$$

with

$$
B_{ \pm}=\frac{3 \mp 1}{6}\left[ \pm 11+\kappa_{ \pm}\right]
$$

and all other quantities defined before. The $\mu$ and scheme dependences of $\xi_{i}^{\mathrm{NF}}$ can in principle be found by using the dependences of $C_{i}(\mu)$ given above and $\varepsilon_{i}(\mu)$ in (27) and (28). To this end, however, one needs the determination of the non-perturbative 
parameters $\varepsilon_{i}(\mu)$ and $\bar{\varepsilon}_{i}(\mu)$ at a single value of $\mu$. If, as done in $\llbracket 7, a_{i}^{\text {eff }}$ are assumed to be universal parameters, the determination of $\varepsilon_{i}(\mu)$ and $\bar{\varepsilon}_{i}(\mu)$ is only possible if one also makes the following universality assumptions:

$$
\varepsilon_{1}(\mu)=\bar{\varepsilon}_{1}(\mu), \quad \varepsilon_{8}(\mu)=\bar{\varepsilon}_{8}(\mu)
$$

In [7] such an assumption was not necessary since $\varepsilon_{1}(\mu), \bar{\varepsilon}_{1}(\mu)$ and $\varepsilon_{8}(\mu)$ were neglected, and only $\bar{\varepsilon}_{8}(\mu)$ was kept in the analysis.

With the assumptions in (37), $\varepsilon_{1}(\mu)$ and $\varepsilon_{8}(\mu)$ can indeed be found once the effective parameters $a_{i}^{\text {eff }}$ have been determined experimentally. Using (10) and (15) together with (37) we find

$$
\begin{gathered}
\varepsilon_{1}(\mu)=\frac{C_{1}(\mu) a_{1}^{\mathrm{eff}}-C_{2}(\mu) a_{2}^{\mathrm{eff}}}{C_{1}^{2}(\mu)-C_{2}^{2}(\mu)}-1 \\
\varepsilon_{8}(\mu)=\frac{a_{2}^{\mathrm{eff}}}{C_{1}(\mu)}-\left(\frac{C_{2}(\mu)}{C_{1}(\mu)}+\frac{1}{N}\right)\left[1+\varepsilon_{1}(\mu)\right]
\end{gathered}
$$

On the other hand $\xi_{i}^{\mathrm{NF}}(\mu)$ can be determined without the universality assumption (37) from two decays simply as follows

$$
\xi_{1}^{\mathrm{NF}}(\mu)=a_{1}^{\mathrm{eff}}-a_{1}(\mu) \quad \xi_{2}^{\mathrm{NF}}(\mu)=a_{2}^{\mathrm{eff}}-a_{2}(\mu)
$$

We will analyze the formulae (38), (39) and (40) in the next section.

The formulae in (40) make it clear that the strict factorization in which $\xi_{i}^{\mathrm{NF}}(\mu)$ vanish can be at best correct at a single value of $\mu$, the so-called factorization scale $\mu_{f}$. In the first studies of factorization $\mu_{f}=m_{\mathrm{b}}$ has been assumed. It has been concluded that such a choice is not in accord with the data [3, 10, 4].

The idea of the generalized factorization as formulated by Neubert and Stech [7] (see also [5, 6] for earlier presentations) is to allow $\mu_{f}$ to be different from $m_{\mathrm{b}}$ and to extract first the non-factorizable parameters $\varepsilon_{i}\left(m_{\mathrm{b}}\right)$ from the data. Subsequently the factorization scale $\mu_{f}$ can be found by requiring these parameters to vanish.

In the numerical analysis of this procedure done in [7] a further assumption has been made. Using large $N$ arguments it has been argued that $\varepsilon_{1}(\mu)$ can be set to zero while $\varepsilon_{8}(\mu)$ can be sizable. The resulting expressions for $a_{i}^{\text {eff }}$ are then

$$
a_{1}^{\mathrm{eff}}=C_{1}\left(m_{\mathrm{b}}\right), \quad a_{2}^{\mathrm{eff}}=a_{2}\left(m_{\mathrm{b}}\right)+C_{1}\left(m_{\mathrm{b}}\right) \varepsilon_{8}\left(m_{\mathrm{b}}\right)
$$

where additional small terms have been dropped in order to obtain the formula for $a_{1}^{\text {eff }}$. Using subsequently the extracted value $a_{2}^{\text {eff }}=0.21 \pm 0.05$ together with the 
coefficients $C_{i}\left(m_{\mathrm{b}}\right)$ from [16] one finds $\varepsilon_{8}\left(m_{\mathrm{b}}\right)=0.12 \pm 0.05$ [7]. Next assuming $\varepsilon_{8}\left(\mu_{f}\right)=0$ one can find the factorization scale $\mu_{f}$ by inverting the formula

$$
\varepsilon_{8}\left(m_{\mathrm{b}}\right)=-\frac{4 \alpha_{s}\left(m_{\mathrm{b}}\right)}{3 \pi} \ln \frac{m_{\mathrm{b}}}{\mu_{f}}
$$

which follows from (28) with $\varepsilon_{8}\left(\mu_{f}\right)=0$ and $\varepsilon_{1}\left(m_{\mathrm{b}}\right)=0$. Thus

$$
\mu_{f}=m_{\mathrm{b}} \exp \left[\frac{3 \pi \varepsilon_{8}\left(m_{\mathrm{b}}\right)}{4 \alpha_{s}\left(m_{\mathrm{b}}\right)}\right] .
$$

Taking $m_{\mathrm{b}}=4.8 \mathrm{GeV}$ and $\alpha_{s}\left(m_{b}\right)=0.21$ (corresponding to $\alpha_{s}\left(M_{\mathrm{Z}}\right)=0.118$ ) we find using $\varepsilon_{8}\left(m_{\mathrm{b}}\right)=0.12 \pm 0.05$ a rather large factorization scale $\mu_{f}=\left(15.9_{-6.6}^{+11.3}\right) \mathrm{GeV}$, roughly a factor of 3-4 higher than $m_{\mathrm{b}}$. This implies that non-factorizable contributions in hadronic matrix elements at scales close to $m_{\mathrm{b}}$ are sizable. This is also signaled by the value of $\varepsilon_{8}\left(m_{\mathrm{b}}\right) \approx 0.12$ which is as large as the factorizable contribution $a_{2}\left(m_{\mathrm{b}}\right)=0.10$ to the effective parameter $a_{2}^{\text {eff }}=0.21 \pm 0.05$.

We would like to emphasize that such an interpretation of the analysis of Neubert and Stech [7] would be misleading. As stressed in [1] the coefficient $a_{2}(\mu)$ is very strongly dependent on the renormalization scheme. Consequently for a given value of $a_{2}^{\text {eff }}$ also $\xi_{2}^{N F}\left(m_{\mathrm{b}}\right)$ and $\varepsilon_{8}\left(m_{\mathrm{b}}\right)$ are strongly scheme dependent. This shows [11, that a meaningful analysis of the $\mu$-dependences in non-leptonic decays, such as the search for the factorization scale $\mu_{f}$, cannot be be made without simultaneously considering the scheme dependence. This is evident if one recalls that any variation of $\mu_{f}$ in the leading logarithm is equivalent to a shift in constant non-logarithmic terms. The latter represent NLO contributions in the renormalization group improved perturbation theory and must be included for a meaningful extraction of $\mu_{f}$ or any other scale like $\Lambda_{\overline{M S}}$. However, once the NLO contributions are taken into account, the renormalization scheme dependence enters the analysis and consequently the factorization scale $\mu_{f}$ at which the non-factorizable hadronic parameters $\xi_{i}^{N F}\left(\mu_{f}\right)$ or $\varepsilon_{i}\left(\mu_{f}\right)$ vanish is renormalization scheme dependent.

From this discussion it becomes clear that for any chosen scale $\mu_{f}=\mathcal{O}\left(m_{\mathrm{b}}\right)$, it is always possible to find a renormalization scheme for which

$$
\xi_{1}^{N F}\left(\mu_{f}\right)=\xi_{2}^{N F}\left(\mu_{f}\right)=0
$$

Indeed as seen in (40) $\xi_{i}^{N F}(\mu)$ depend through $a_{i}(\mu)$ on $\kappa_{ \pm}$which characterize a given renormalization scheme. The choice of $\kappa_{ \pm}$corresponds to a particular finite 
renormalization of the operators $O_{ \pm}$in addition to the renormalization in the NDR scheme. It is then straightforward to find the values of $\kappa_{ \pm}$which assure that for a chosen scale $\mu_{f}$ the conditions in (44) are satisfied. We find

$$
\begin{gathered}
\kappa_{+}=3\left[\frac{3}{4} \frac{a_{1}^{\mathrm{eff}}+a_{2}^{\mathrm{eff}}}{W_{+}\left(\mu_{f}\right)}-1\right] \frac{4 \pi}{\alpha_{s}\left(\mu_{f}\right)}-3\left(J_{+}\right)_{\mathrm{NDR}}, \\
\kappa_{-}=\frac{3}{2}\left[\frac{3}{2} \frac{a_{1}^{\mathrm{eff}}-a_{2}^{\mathrm{eff}}}{W_{-}\left(\mu_{f}\right)}-1\right] \frac{4 \pi}{\alpha_{s}\left(\mu_{f}\right)}-\frac{3}{2}\left(J_{-}\right)_{\mathrm{NDR}},
\end{gathered}
$$

where

$$
W_{ \pm}\left(\mu_{f}\right)=\left[\frac{\alpha_{s}\left(M_{W}\right)}{\alpha_{s}\left(\mu_{f}\right)}\right]^{d_{ \pm}}\left[1+\frac{\alpha_{s}\left(M_{W}\right)}{4 \pi}\left(B_{ \pm}-J_{ \pm}\right)\right]
$$

with $\left(J_{ \pm}\right)_{\text {NDR }}$ being the values of $J_{ \pm}$in the NDR scheme. $W_{ \pm}\left(\mu_{f}\right)$ are clearly renormalization scheme independent as $B_{ \pm}-J_{ \pm}$are scheme independent.

\section{Numerical Analysis}

Before presenting the numerical analysis of the formulae derived in the preceding section, we would like to clarify the difference between the Wilson coefficients in (34) and (35) used by us and the ones employed in [7]. In [7] the scheme independent coefficients $\tilde{z}_{ \pm}(\mu)$ of [16] instead of $z_{ \pm}(\mu)$ have been used. These are obtained by multiplying $z_{ \pm}(\mu)$ by $\left(1-B_{ \pm} \alpha_{s}(\mu) / 4 \pi\right)$ so that

$$
\tilde{z}_{ \pm}(\mu)=\left[\frac{\alpha_{s}\left(M_{W}\right)}{\alpha_{s}(\mu)}\right]^{d_{ \pm}}\left[1+\frac{\alpha_{s}\left(M_{W}\right)-\alpha_{s}(\mu)}{4 \pi}\left(B_{ \pm}-J_{ \pm}\right)\right] .
$$

These coefficients are clearly not the coefficients of the operators $O_{ \pm}$used in [7] and here. In order to be consistent the matrix elements $\left\langle O_{ \pm}\right\rangle$should then be replaced by

$$
\left\langle\tilde{O}_{ \pm}\right\rangle=\left(1+B_{ \pm} \alpha_{s}(\mu) / 4 \pi\right)\left\langle O_{ \pm}\right\rangle
$$

This explains why the results of our numerical analysis differ considerably from the ones presented in [7]. We strongly advice the practitioners of non-leptonic decays not to use the scheme independent coefficients of [16] in phenomenological applications. These coefficients have been introduced to test the compatibility of different renormalization schemes and can only be used for phenomenology together with $\left\langle\tilde{O}_{ \pm}\right\rangle$. This would however unnecessarily complicate the analysis and it is therefore advisable to work with the true coefficients $C_{i}(\mu)$ of the operators $O_{i}$ as given in (34) and (35). 


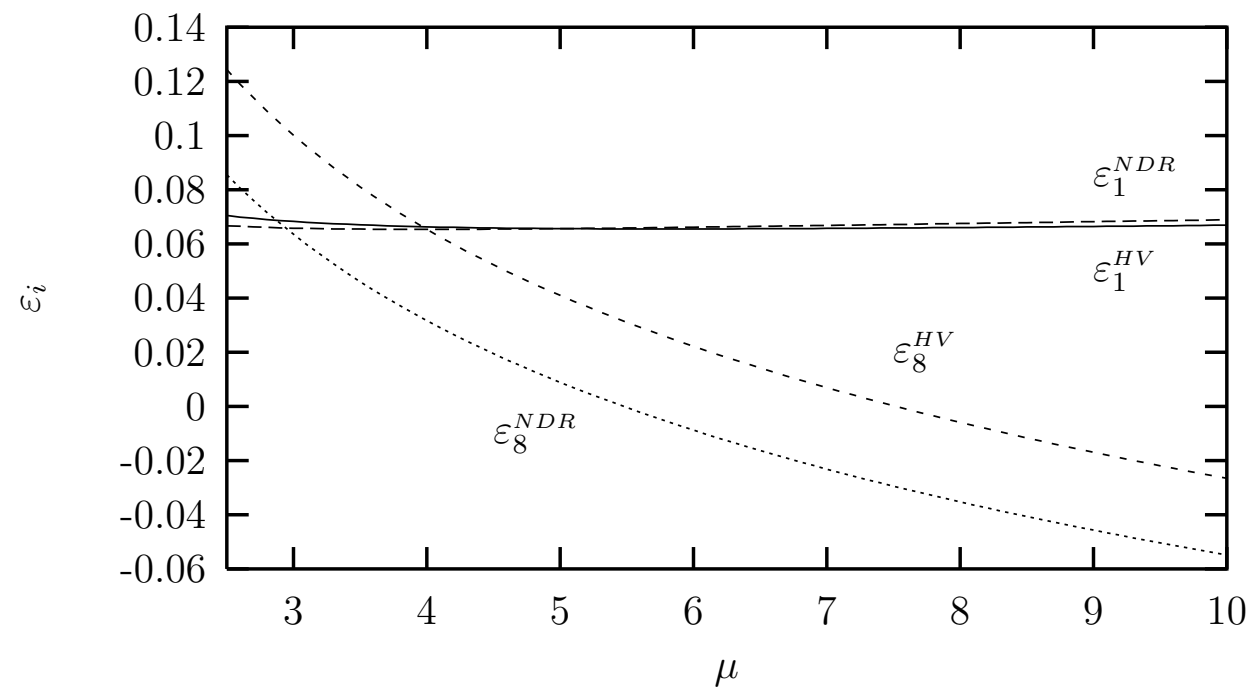

Figure 1: $\varepsilon_{1,8}(\mu)$ in the NDR and HV schemes.

In [7] the following values of $a_{i}^{\text {eff }}$ have been extracted from existing data on twobody B-decays

$$
a_{1}^{\mathrm{eff}}=1.08 \pm 0.04 \quad a_{2}^{\mathrm{eff}}=0.21 \pm 0.05
$$

with similar results given in [5, 6, 10, 8, 9]. In order to illustrate various points made in the preceding section, we take the central values of $a_{i}^{\text {eff }}$ in (50). Using (38)-(40) we calculate $\varepsilon_{i}(\mu)$ and $\xi_{i}^{N F}(\mu)$ as a function of $\mu$ in the range $2.5 \mathrm{GeV} \leq \mu \leq 10 \mathrm{GeV}$ for the NDR and HV schemes. The results are shown in fig. 11 and fig. 2. We observe that $\varepsilon_{1}(\mu)$ and $\xi_{1}^{\mathrm{NF}}(\mu)$ are only weakly $\mu$ and scheme dependent in accordance with the findings in [11], where these dependences have been studied for $a_{i}(\mu)$ defined in (11). The strong $\mu$ and scheme dependences of $a_{2}(\mu)$ found there translate into similar strong dependences of $\varepsilon_{8}(\mu)$ and $\xi_{2}^{\mathrm{NF}}(\mu)$.

We make the following observations:

- $\varepsilon_{1}(\mu)$ and $\xi_{1}^{\mathrm{NF}}(\mu)$ are non-zero in the full range of $\mu$ considered.

- $\varepsilon_{8}(\mu)$ and $\xi_{2}^{\mathrm{NF}}(\mu)$ vary strongly with $\mu$ and vanish in the NDR scheme for $\mu=5.5 \mathrm{GeV}$ and $\mu=6.3 \mathrm{GeV}$ respectively. The corresponding values in the HV scheme are $\mu=7.5 \mathrm{GeV}$ and $\mu=8.6 \mathrm{GeV}$.

- There is no value of $\mu=\mu_{f}$ in the full range considered for which $\varepsilon_{1}(\mu)$ and 


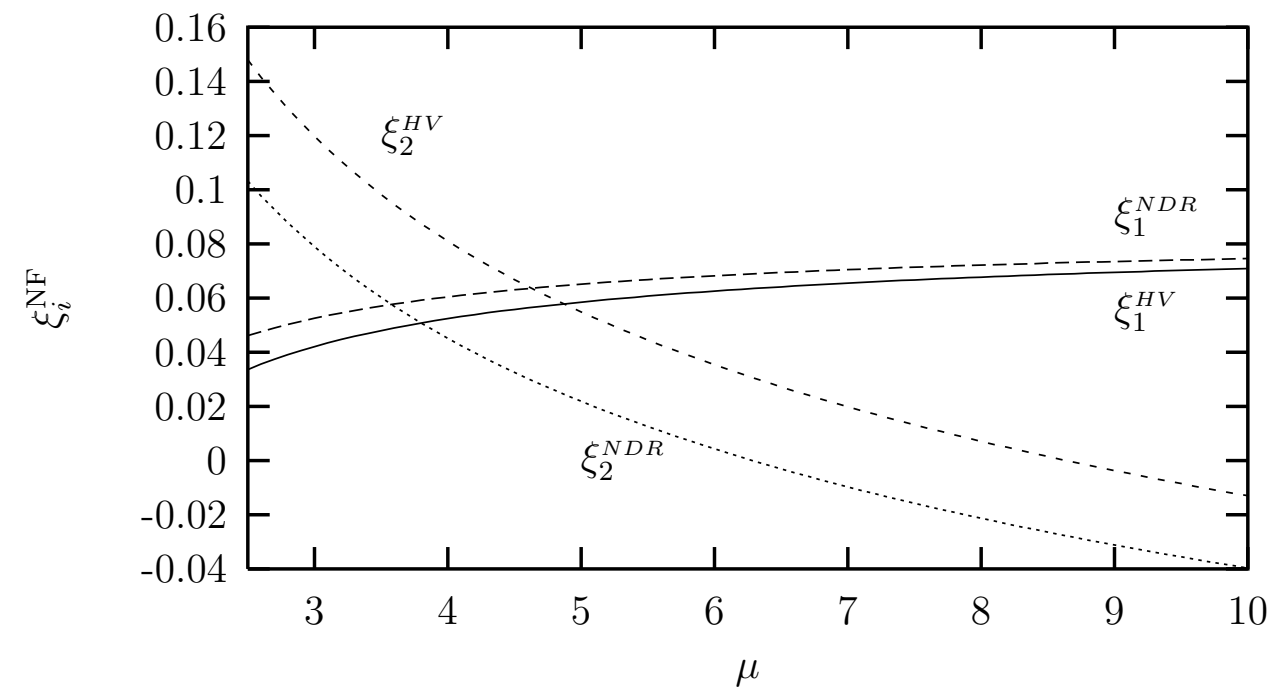

Figure 2: $\xi_{1,2}^{\mathrm{NF}}(\mu)$ in the NDR and HV schemes.

$\varepsilon_{8}(\mu)$ or equivalently $\xi_{1}^{\mathrm{NF}}(\mu)$ and $\xi_{2}^{\mathrm{NF}}(\mu)$ simultaneously vanish. We also observe contrary to expectations in [7] that $\varepsilon_{1}(\mu)$ is not necessarily smaller than $\varepsilon_{8}(\mu)$. In fact the large $N$ arguments presented in [7] that $\varepsilon_{1}(\mu)=\mathcal{O}\left(1 / N^{2}\right)$ and $\varepsilon_{8}(\mu)=\mathcal{O}(1 / N)$, imply strictly speaking only that the $\mu$-dependence of $\varepsilon_{8}(\mu)$ is much stronger than that of $\varepsilon_{1}(\mu)$, which we indeed see in figs. 1 1 and 2 . The hierarchy of their actual values is a dynamical question. Even if the large $N$ counting rules $\varepsilon_{1}(\mu)=\mathcal{O}\left(1 / N^{2}\right)$ and $\varepsilon_{8}(\mu)=\mathcal{O}(1 / N)$ are true independently of the factorization hypothesis [20, 21], it follows from our analysis that once the generalized factorization hypothesis is made, the extracted values of $\varepsilon_{i}$ violate for some range of $\mu$ the large- $\mathrm{N}$ rule $\varepsilon_{1} \ll \varepsilon_{8}$.

We can next investigate for which renormalization scheme characterized by $\kappa_{ \pm}$the factorization is exact at $\mu_{f}=m_{\mathrm{b}}=4.8 \mathrm{GeV}$. We call this choice the "factorization scheme" (FS). Using the central values in (50) and $\Lambda \frac{(5)}{M S}=225 \mathrm{MeV}$ we find by means of (45) and (46)

$$
\kappa_{+}=13.5, \quad \kappa_{-}=3.9 \quad(\mathrm{FS}) .
$$

These values deviate considerably from the NDR values $\kappa_{ \pm}=0$ and the HV values $\kappa_{ \pm}=\mp 4$. Yet one can verify that for these values $J_{+}=6.13$ and $J_{-}=1.17$ and consequently in this scheme the NLO corrections at $\mu=m_{\mathrm{b}}$ remain perturbative. In 
Table 1: $\quad \xi_{1,2}^{\mathrm{NF}}(\mu)$ as functions of $\mu$ for different schemes and $\Lambda \frac{(5)}{M S}=225 \mathrm{MeV}$.

\begin{tabular}{|c|c|c|c||c|c|c|}
\hline & \multicolumn{3}{|c||}{$\xi_{1}^{\mathrm{NF}}(\mu)$} & \multicolumn{3}{c|}{$\xi_{2}^{\mathrm{NF}}(\mu)$} \\
\hline$\mu[\mathrm{GeV}]$ & NDR & HV & FS & NDR & HV & FS \\
\hline \hline 2.5 & 0.046 & 0.035 & -0.033 & 0.102 & 0.144 & 0.075 \\
\hline 5.0 & 0.065 & 0.059 & 0.001 & 0.022 & 0.055 & -0.004 \\
\hline 7.5 & 0.071 & 0.067 & 0.014 & -0.016 & 0.013 & -0.041 \\
\hline 10.0 & 0.074 & 0.071 & 0.021 & -0.039 & -0.013 & -0.064 \\
\hline
\end{tabular}

table 1 we give the values of $\xi_{i}^{\mathrm{NF}}(\mu)$ for the NDR, HV and FS schemes.

The numerical analysis presented here used as input the central values for $a_{i}^{\text {eff }}$ given in (50). As stressed in particular in [22], the strong model dependence of the form factors and large experimental errors preclude at present a precise determination of these parameters. Consequently when these uncertainties are taken into account, the differences between various schemes are washed out to some extent. Yet the general features of the results obtained for other numerical values of the pair $\left(a_{1}^{\text {eff }}, a_{2}^{\text {eff }}\right)$ are very similar to the ones presented here.

\section{$5 \quad$ Generalized Factorization and $N^{\mathrm{eff}}$}

As pointed sometime ago in [13, 23] and recently discussed in [5, 8, 9], it is always possible to calculate the scale and scheme dependence of the hadronic matrix elements in perturbation theory by simply calculating the matrix elements of the relevant operators between the quark states. Combining these scheme and scale dependent contributions with the Wilson coefficients $C_{i}(\mu)$ one obtains the effective coefficients $C_{i}^{\text {eff }}$ which are free from these dependences. If one neglects in addition final state interactions and other possible non-factorizable contributions the decay amplitudes can be generally written as follows

$$
A=\left\langle H_{\mathrm{eff}}\right\rangle=\frac{G_{F}}{\sqrt{2}} V_{C K M}\left[C_{1}^{\mathrm{eff}}\left\langle O_{1}\right\rangle^{\text {tree }}+C_{2}^{\mathrm{eff}}\left\langle O_{2}\right\rangle^{\text {tree }}\right]
$$

where $\left\langle O_{i}\right\rangle^{\text {tree }}$ denote tree level matrix elements. The proposal in [5, 8, 9] is to use (52) and to apply the idea of the factorization to the tree level matrix elements. In this approach then the effective parameters $a_{1,2}^{\text {eff }}$ are given by (5) with $N^{\text {eff }}$ treated as 
a phenomenological parameter which models those non-factorizable contributions to the hadronic matrix elements, which have not been included into $C_{i}^{\text {eff }}$. In particular it has been suggested in [5, 8, 9] that the values for $N^{\text {eff }}$ extracted from the data on two-body non-leptonic decays should teach us about the pattern of non-factorizable contributions.

In particular when calculating the effective coefficients $C_{i}^{\text {eff }}$, the authors of [8, 9] have included a subset of contributions to the perturbative matrix elements, which is sufficient to cancel the scale and scheme dependence of the Wilson coefficients. Unfortunately the results of such calculations are generally gauge dependent and suffer from the dependence on the infrared regulator and generally on the assumptions about the external momenta.

Let us discuss this point in detail, following [23]. The Green function of the renormalized operator $O$, for a given choice of the ultraviolet regularization (NDR or $\mathrm{HV}$ for example), a choice of the external momenta $p$ and of the gauge parameter $\lambda$, is given by

$$
\Gamma_{O}^{\lambda}(p)=1+\frac{\alpha_{s}}{4 \pi}\left(-\frac{\gamma^{(0)}}{2} \ln \left(\frac{-p^{2}}{\mu^{2}}\right)+\hat{r}\right)
$$

with

$$
\hat{r}=\hat{r}^{N D R, H V}+\lambda \hat{r}^{\lambda}
$$

The matrices $\hat{r}^{N D R, H V}$ depend on the choice of the external momenta and on the ultraviolet regularization, while $\hat{r}^{\lambda}$ is regularization- and gauge-independent, but depends on the external momenta. It is clearly possible to define a renormalization scheme in which, for given external momenta and gauge parameter, $\Gamma_{O}^{\lambda}(p)=1$, or in other words $\langle O\rangle_{p, \lambda}=\langle O\rangle^{\text {tree }}$ (this corresponds to the RI scheme discussed in [23]). However, the definition of the renormalized operators will now depend on the choice of the gauge and of the external momenta. If one were able, for example by means of lattice QCD, to compute the matrix element of the operator using the same renormalization prescription, the dependences on the gauge and on the external momenta would cancel between the Wilson coefficient and the matrix element. If, on the contrary, the matrix elements are estimated using factorization, no trace is kept of the renormalization prescription and the final result is gauge and infrared dependent.

In [8, 9] scale- and scheme-independent effective Wilson coefficients have been obtained by adding to $C_{i}(\mu)$ the contributions coming from vertex-type quark matrix 
elements, denoted by $\hat{r}_{V}$ and $\hat{\gamma}_{V}$. In particular

$$
\begin{aligned}
& C_{1}^{\mathrm{eff}}=C_{1}(\mu)+\frac{\alpha_{s}}{4 \pi}\left(r_{V}^{T}+\gamma_{V}^{T} \log \frac{m_{b}}{\mu}\right)_{1 j} C_{j}(\mu), \\
& C_{2}^{\mathrm{eff}}=C_{2}(\mu)+\frac{\alpha_{s}}{4 \pi}\left(r_{V}^{T}+\gamma_{V}^{T} \log \frac{m_{b}}{\mu}\right)_{2 j} C_{j}(\mu) .
\end{aligned}
$$

where the index $j$ runs through all contributing operators, also penguin operators considered in [5, 8, 9].

It is evident from the above discussion that $\hat{r}_{V}$ depends not only on the external momenta, but also on the gauge chosen. For example, in [8, 9] the following result for $\hat{r}_{V}$ is quoted:

$$
\hat{r}_{V}=\left(\begin{array}{cccccc}
\frac{7}{3} & -7 & 0 & 0 & 0 & 0 \\
-7 & \frac{7}{3} & 0 & 0 & 0 & 0 \\
0 & 0 & \frac{7}{3} & -7 & 0 & 0 \\
0 & 0 & -7 & \frac{7}{3} & 0 & 0 \\
0 & 0 & 0 & 0 & -\frac{1}{3} & 1 \\
0 & 0 & 0 & 0 & -3 & \frac{35}{3}
\end{array}\right) .
$$

This result is valid in the Landau gauge $(\lambda=0)$; in an arbitrary gauge, with the same choice of external momenta used to obtain (56) one would get

$$
\hat{r}_{V}=\hat{r}_{V}(\lambda=0)+\lambda r_{V}^{\lambda},
$$

with $\hat{r}_{V}(\lambda=0)$ given in (56) and

$$
r_{V}^{\lambda}=\left(\begin{array}{cccccc}
-\frac{5}{6} & -\frac{3}{2} & 0 & 0 & 0 & 0 \\
-\frac{3}{2} & -\frac{5}{6} & 0 & 0 & 0 & 0 \\
0 & 0 & -\frac{5}{6} & -\frac{3}{2} & 0 & 0 \\
0 & 0 & -\frac{3}{2} & -\frac{5}{6} & 0 & 0 \\
0 & 0 & 0 & 0 & -\frac{11}{6} & \frac{3}{2} \\
0 & 0 & 0 & 0 & 0 & \frac{8}{3}
\end{array}\right) .
$$

The expressions for the full $10 \times 10 \hat{r}$ matrices in the NDR and HV schemes and in the Feynman and Landau gauges are given in [23], for a different choice of the external momenta. The results for the Landau gauge are given in [13].

Equation (57) shows that the definition of the effective coefficients advocated in

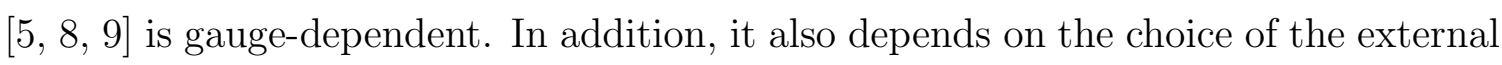


momenta. This implies that the effective number of colors extracted in [5, 8, 9] is also gauge-dependent, and therefore it cannot have any physical meaning.

The gauge dependences and infrared dependences discussed here are not new. They appear in any calculation of matrix elements of operators between quark states necessary in the process of matching of the full theory onto an effective theory. A particular example can be found in [24] where the full gauge dependence of the quark matrix element of the operator $(\bar{s} d)_{V-A}(\bar{s} d)_{V-A}$ has been calculated. However, in the process of matching such unphysical dependences in the effective theory are cancelled by the corresponding contributions in the full theory so that the Wilson coefficients are free of such dependences. Similarly in the case of inclusive decays of heavy quarks, where the spectator model can be used, they are cancelled by gluon bremsstrahlung. In exclusive hadron decays there is no meaningful way to include such effects in a perturbative framework and one is left with the gauge and infrared dependences in question.

\section{Summary}

In this paper we have critically analyzed the hypothesis of the generalized factorization. While the parametrization of the data in terms of a set of effective parameters discussed in [5- 9] may appear to be useful, we do not think that this approach offers convincing means to analyze the physics of non-factorizable contributions to non-leptonic decays. In particular:

- The renormalization scheme dependence of the non-factorizable contributions to hadronic matrix elements precludes the determination of the factorization scale $\mu_{f}$.

- Consequently for any chosen value of $\mu_{f}=\mathcal{O}\left(m_{b}\right)$ it is possible to find a renormalization scheme for which the non-perturbative parameters $\varepsilon_{1,8}$ used in [7] to characterize the size of non-factorizable contributions vanish. The same applies to $\xi_{1,2}^{\mathrm{NF}}(\mu)$ introduced in the present paper.

- We point out that the recent extractions of the effective number of colours $N^{\text {eff }}$ from two-body non-leptonic B-decays, presented in [5, 8, 9], while $\mu$ and renormalization scheme independent suffer from gauge dependences and infrared regulator dependences. 
A further problem in the generalized factorization approach is given by the presence in many channels of operators that contribute only through non-factorizable terms. These contributions cannot be incorporated in the definitions of $\varepsilon_{1}$ and $\varepsilon_{8}$, and a more general parametrization is needed [25]. A typical example is given by charming-penguin contributions to $B \rightarrow K \pi$ decays [26].

We hope that our analysis demonstrates clearly the need for an approach to nonleptonic decays which goes beyond the generalized factorization discussed recently in the literature. Some possibilities are offered by dynamical approaches like QCD sum rules as recently reviewed in [27]. However, even a phenomenological approach which does not suffer from the weak points of factorization discussed here, would be a step forward. We hope to present some ideas in this direction in a forthcoming publication 28.

\section{References}

[1] J. Schwinger, Phys. Rev. Lett. 12 (1964) 630; R.P. Feynman, in Symmetries in Particle Physics, ed. A. Zichichi, Acad. Press 1965, p.167; O. Haan and B. Stech, Nucl. Phys. B 22 (1970) 448.

[2] D. Fakirov and B. Stech, Nucl. Phys. B 133 (1978) 315; L.L. Chau, Phys. Rep. 95 (1983) 1.

[3] M. Wirbel, B. Stech and M. Bauer, Z. Phys. C 29 (1985) 637; M. Bauer, B. Stech and M. Wirbel, Z. Phys. C 34 (1987) 103.

[4] M. Neubert, V. Rieckert, B. Stech and Q.P. Xu, in "Heavy Flavours", eds. A.J. Buras and M. Lindner (World Scientific, Singapore, 1992), p. 286;

[5] H.-Y. Cheng, Phys. Lett. B 335 (1994) 428, Phys. Lett. B 395 (1997) 345;

H.-Y. Cheng and B. Tseng, hep-ph/9708211, hep-ph/9803457.

[6] J.M. Soares, Phys. Rev. D 51 (1995) 3518.

[7] M. Neubert and B. Stech, Preprint CERN-TH/97-99, hep-ph/9705292, to appear in Heavy Flavours II, edited by A.J. Buras and M. Lindner (World Scientific, Singapore); B. Stech, hep-ph/9706384; M.Neubert, Nucl. Phys. Proc. Suppl. B 64 (1998) 474, hep-ph/9801269. 
[8] A. Ali and C. Greub, Phys. Rev. D57 (1998) 2996; A. Ali, J. Chay, C. Greub and P. Ko, Phys. Lett. B 424 (1998) 161.

[9] A. Ali, G. Kramer and C.-D. Lü, hep-ph/9804363.

[10] D. Du and Z. Xing, Phys. Lett. B 312 (1993) 199; A. Deandrea et al., Phys. Lett. B 318 (1993) 549, Phys. Lett. B 320 (1994) 170; N.G. Deshpande, B. Dutta, S. Oh, Phys. Rev. D 57 (1998) 5723, hep-ph/9712445.

[11] A.J. Buras, Nucl. Phys. B 434 (1995) 606.

[12] A.J. Buras, M. Jamin, and M.E. Lautenbacher, Nucl. Phys. B 408 (1993) 209.

[13] A.J. Buras, M. Jamin, M.E. Lautenbacher and P.H. Weisz, Nucl. Phys. B 370 (1992) 69, Nucl. Phys. B 400 (1993) 37.

[14] A.J. Buras, M. Jamin and M.E. Lautenbacher, Nucl. Phys. B 400 (1993) 75.

[15] M. Ciuchini, E. Franco, G. Martinelli and L. Reina, Phys. Lett. B 301 (1993) 263, Nucl. Phys. B 415 (1994) 403.

[16] A.J. Buras and P.H. Weisz, Nucl. Phys. B 333 (1990) 66.

[17] G. Altarelli, G. Curci, G. Martinelli and S. Petrarca, Nucl. Phys. B 187 (1981) 461.

[18] W.A. Bardeen, A.J. Buras, D.W. Duke and T. Muta, Phys. Rev. D 18 (1978) 3998.

[19] M. Schmelling, in proceedings of the 28th International Conference on High Energy Physics, July 1996, Warsaw, Poland, page 91.

[20] E. Witten, Nucl. Phys. B 160 (1979) 57.

[21] A.J. Buras, J.M. Gèrard and R. Rückl, Nucl. Phys. B 268 (1986) 16.

[22] M. Ciuchini, R. Contino, E. Franco, G. Martinelli and L. Silvestrini, hep$\mathrm{ph} / 9801420$.

[23] M. Ciuchini, E. Franco, G. Martinelli, L. Reina and L. Silvestrini, Z.Phys. C68 (1995) 239. 
[24] A.J. Buras, M. Jamin, and P.H. Weisz, Nucl. Phys. B 347 (1990) 491.

[25] M. Ciuchini, E. Franco, G. Martinelli and L. Silvestrini, Nucl. Phys. B 501 (1997) 271.

[26] M. Ciuchini, R. Contino, E. Franco, G. Martinelli and L. Silvestrini, Nucl. Phys. B $512(1998) 3$.

[27] A. Khodjamirian and R. Rückl, hep-ph/9801443, to appear in Heavy Flavours II, edited by A.J. Buras and M. Lindner (World Scientific, Singapore).

[28] A.J. Buras and L. Silvestrini, work in progress. 